\title{
Incredibly Versatile Microbial Fuel Cells Innovative Ideas at HES-SO Valais-Wallis for Solving Topical Problems
}

\section{Elsbeth Heinzelmann, Journalist Science + Technology}

Abstract: At HES-SO Valais-Wallis, Prof. Fabian Fischer is specialized in microbial fuel cells for novel applications that meet the challenge of producing renewable energies. He and his team possess a unique expertise in bioelectric energy vector generation, phosphate extraction (CHIMIA 2015, 69, 296) and the testing of antimicrobial surfaces. Let's take a look behind the scenes of the Institute of Life Technologies in Sion.

Keywords: Antimicrobial surface - Cicada Psaltoda claripennis · Methanogenesis · Microbial Electrolysis/Fuel Cells · Nanostructured gold surfaces · Organic substrates · Water treatment

Experts have known all along: in the laboratory of Prof. Fabian Fischer, the development of Microbial Fuel Cells (MFCs) for power production from biomass has become a key activity. MFCs are electrochemical devices that use microbes to oxidize organic matter while, at the same time, generating electricity.

\section{MFCs - A Source of Power}

The fact that MFCs exploit bacterial metabolism to produce electrical current from a wide variety of organic substrates makes researchers around the world prick up their ears. Intense research is under way as much work remains to be done: To date, only a few marine sediment MFCs can be used in practice as they are the only ones capable of providing current for low power devices. "We need to perfect the MFC technology by deepening our understanding of the limitations and the microbiology of these components. The focus of current research is on the scaleup of MFC and Microbial Electrolysis Cell (MEC), which is not yet fully understood", explains Fabian Fischer. "We have now reached the level of a 10-30 litre reactor. Incidentally, it seems that producing electricity is not the only essential factor, but rather the capacity of the electrode-associated microbes to degrade wastes and toxic chemicals. We now know that we need a whole range of options for alternative energy sources to bridge the gap left by exhaustible fossil fuels. Therefore, the discovery of Shewanella oneidensis or Geobacter sulfurreducens as exoelectrogenics in MFCs was a key development. Both are metal-

Stacked 12-litre microbial fuel cell for producing electricity. This forms the basic unit for a modular extendable construction for scale-up research (Source: Fabian Fischer, HES-SO Valais-Wallis).

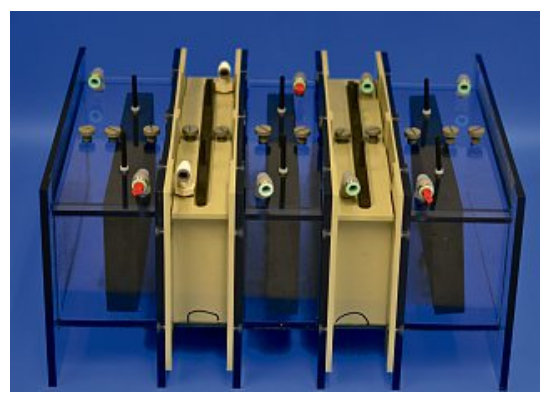

reducing bacteria capable of high current production, and they had attracted extensive international attention a few years ago."

Devices powered by MFC technology have - for instance proved successful in practice inside meteorological buoys. These instruments measure air temperature, pressure, relative humidity and water temperature. Their data are transferred in real-time by radio frequency telemetry powered by Benthic Microbial Fuel Cells (BMFCs). Such BMFCs present a novel form of energy harvesting for marine environments. They have been operating for several years with no decrease in power output. Experts speculate that BMFCs could provide power indefinitely at the same power levels delivered by a lead acid battery for one year. "Currently, MFCs are not yet commercialized, although they are very promising for water treatment and as power sources for environmental sensors", says Fabian Fischer. "The reason for the still limited power generation is the high internal ohmic - resistance. But we are working on improving the system architecture and will soon achieve a power generation that is dependent solely on the efficiency of the microorganisms."

\section{Powerful Methane Storage}

As the end of fossil fuels looms, new ideas and concepts are required. Biofuels are highly rated among experts in the field. Biohydrogen in particular - hydrogen produced via biological processes - is enjoying great popularity. Hydrogen produces electricity as needed in a clean and highly efficient way in chemical fuel cells. During the past 20 years, scientists have focused on two bioprocesses: on the one hand we have the conversion of carbohydrates to hydrogen by fermentative bacteria. This so-called dark fermentation has its limits, as the yield is very low. On the other hand we have the conversion of organic acids to hydrogen by photosynthetic bacteria. This so-called photo-fermentation makes use of organic acids and increases the overall hydrogen yield. However, the process requires light, which complicates the reactor design and renders any large-scale application impossible.

The method for producing hydrogen or methane from biomass - biological material derived from living or recently living organisms - by microbial electrolysis cell technology has opened up new avenues for improving current methanisation well beyond the state of the art. The process takes place in a bio-electrochemically assisted microbial reactor known as the microbial electrolysis cell (MEC). Electrochemically active microbes on the surface of the anode break down organic matter into $\mathrm{CO}_{2}$, electrons and protons. The electrons pass through the

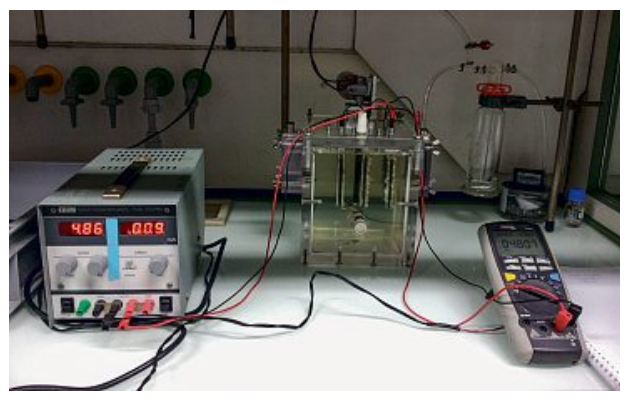

Microbial electrolysis cell for biohydrogen generation with incoming wastewater (Source: Fabian Fischer, HES-SO Valais-Wallis). 
external circuit while the protons migrate internally to finally combine at the cathode and evolve as hydrogen gas. "MEC is a promising production system for renewable energy stored in the form of hydrogen or methane", states Fabian Fischer, who formerly worked at the University of California at Berkeley and at the Pierre and Marie Curie University in Paris. "Usually organic matter in sewage or municipal solid waste is microbiologically converted mainly into methane and $\mathrm{CO}_{2}$ under anaerobic conditions. By applying a low electrical current the composition of the gas can be shifted towards a higher yield of methane and a reduction in $\mathrm{CO}_{2}$."

\section{Discover the Positive Aspects of Methane}

But isn't methane a greenhouse gas that raises the earth's temperature? The scientist provides clarification: "Methane can easily be contained in reactors and harnessed as an energy source." $\mathrm{He}$ describes methanogenesis as an anaerobic fermentation process involving low-cost substrates such as sewage transformed into methane. The fermentation itself can be divided in four single steps in which different consortia of microorganisms perform the transformation: hydrolysis of organic material into monomers, acidogenesis, acetogenesis and methanogenesis. As Fabian Fischer tells us, electromethanogenesis was originally an undesired side reaction in single-chamber MECs. Instead of focusing exclusively on suppressing the electromethanogenesis, it was used to produce more methane than was possible with traditional methane-producers. Storability and transportation are better for methane than for hydrogen: they are widely implemented because methane is also the main component of natural gas. Therefore, the existing infrastructure can be used. Furthermore, a main advantage lies in its ability to capture carbon dioxide, for example from industrial emissions or anaerobic digesters. "Although methane has a higher global warming potential as carbon dioxide, it offers a very efficient form of energy storage and is easier to handle than hydrogen", says Fabian Fischer. At his laboratory he and his team are developing MEC in which low-cost substrates such as municipal sewage and fermentation waste are used as sources to generate methane or hydrogen. "In conclusion, MEC technology has the potential to become a viable solution for energy and environmental problems."

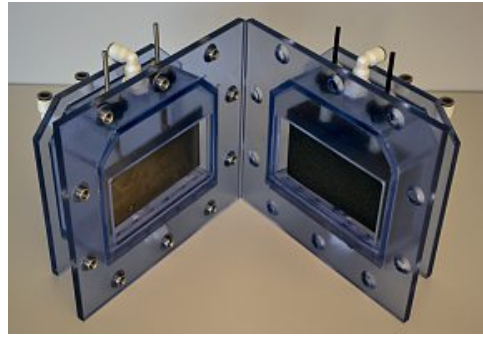

Microbial electrolysis cell useful for hydrogen and methane generation (Source: Fabian Fischer, HES-SO Valais-Wallis).

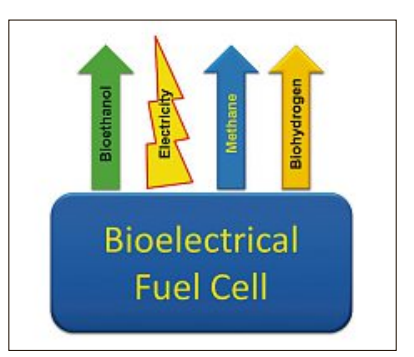

Some of the possible energy vectors that can be produced with microbial fuel cell type reactors (Source: Fabian Fischer, HES-SO Valais-Wallis).

\section{The Mystery of Antimicrobial Surfaces}

In the novel domain of antimicrobial surface properties of medical implants, Fabian Fischer and his team are performing pioneering work in the pre-project BugBanSensor - supported by biotechnet - regarding antimicrobial surface testing. As recent research shows, the cicada Psaltoda claripennis from Eastern
Australia has wings that are particularly interesting for biomedical applications. Due to their special nanostructure, these wings have antibacterial properties. On one hand they are superhydrophobic while, on the other, they comprise a lot of nano-pillars. Thanks to their superhydrophobic surfaces they reduce bacterial adhesion. Consequently, the cicada's wings show efficient bactericidal and anti-biofouling effects that we could exploit for medical implants, where the high risk of infections is always hanging like the sword of Damocles over patients as they recover from surgery.

"Currently, there are no suitable standardized methods for biofilm detection and quantification on implantable devices, probably because of the complexity of various biofilm structures and compositions", explains Fabian Fischer. "We lack the materials that offer the required functional properties." He and his collaborators considered whether or not such biofilms adhere on potentially antimicrobial nanosurfaces and are functional or whether they possess antimicrobial properties that can be analysed within a MFC. To this end, nano surfaces have to be made conductive by sputtering them with a conductive material, or else have to be fabricated entirely from conductive material. Such antimicrobial nano-pillars can be synthesized using nanoimprint lithography or a templated electrodeposition technique. Although metal surfaces are rarely used with MFC due to passivation issues, there is one exception: Gold $(\mathrm{Au})$ is highly stable when in contact with biological systems. "This stability is due to its electrode standard potential of $1.69 \mathrm{~V}$, which makes it inert in a microbial environment" explains the scientist, recalling that flat gold surfaces in microbial fuel cells were found to be recalcitrant to biofilm adhesion. "To circumvent this problem, we produced nanostructures from gold to improve biofilm adhesion and tested thereby if they clearly enhanced MFC performance." If the surface has a certain roughness this has beneficial effects for biofilm adhesion to gold.

\section{New Ground for Surface Properties}

As shown by the resulting tests, the nanostructured gold surfaces achieved surprisingly high power density maxima up to $1910 \mathrm{~mW} / \mathrm{m}^{2}$, while flat surfaces on the nanoscale provided almost no power, at just $0.35 \mathrm{~mW} / \mathrm{m}^{2}$. The scientists made sure that the antimicrobial nano-surfaces were covered with a biofilm by adding ampicillin. The antibiotic's bactericidal effect was demonstrated by oscillating and declining potentiometric signals. Fabian Fischer looks back on a very successful development: "The microbial fuel cell-based sensor is a novel tool for evaluating the antimicrobial effects caused by both nano-surfaces and antibiotics."

The range of applications for anti-microbial surfaces is far from being limited to the biomedical field: There is considerable demand in the food and textile industries for enhanced safety benefits. This extends to the protection of food from microbial contamination in order to prevent food poisoning and the production of clothes with anti-microbial properties so as to avoid contamination during processing by employees. "Nano-surface analysis is a new domain. On the one hand it opens up the testing of antimicrobial surfaces and the effectiveness of antibiotics against resistant bacteria and, on the other, it is of great interest to biointerface research. The aim is to develop more efficient MFC electrodes capable of offering considerable benefits in all energy applications for MFC." He smiles and concludes: "In this domain we are only at the start of a long and challenging process. This will be exciting...!"

E-mail: fabian.fischer@hevs.ch

URL: www.hevs.ch

Received: May 6, 2016 